\title{
MobiSpell: Educational Mobile Game Design and Development for Teaching Spelling to Young Children
}

\author{
Menelaos Bakopoulos and Sofia Tsekeridou \\ Athens Information Technology, 0.8km Markopoulou Ave., 19002 Peania, Athens, Greece \\ \{mbak, sots\} aait.edu.gr
}

\begin{abstract}
A 3D educational mobile game is designed and implemented with the aim to teach hard-to-spell words to young children exploiting their natural affinity towards games entertainment in order to increase the likelihood of useful knowledge acquisition while having fun. The educational game features a fighter plane style environment in which spelling is accomplished by shooting down letters. Incentives and motivation such as points and medals encourage learning and motivate children to develop skills. The J2ME Mobile 3D Graphics library is used with 3D models developed in 3DS MAX, and development taking place in Netbeans.
\end{abstract}

Keywords: educational game, mobile game, game design, game development.

\section{Introduction, Goals, and Design Principles}

Rapid technological development has led to modern mobile platforms having equivalent computational power with the early nineties PCs - this has set the grounds to develop entertaining educational mobile games leading it to be a rapidly developing field all over the world.

In this paper, we present an educational mobile game in which a child takes the role of a pilot being tasked with the mission to shoot down various letters and opponents necessary to spell a word correctly. The child is guided by various multimedia cues (such as photos) and blanks representing letters as to which letter should be spelled. In the sequel, we elaborate on key aspects considered in MobiSpell's design referring to deployed technologies and theories, and continue by describing the basic implementation, with conclusions for future work.

MobiSpell's game design has focused on achieving an increased player experience, fun and at the same time learning. Enjoyment directly affects the time spent playing and hence the knowledge retention a child will achieve [1], which made it the primary design principle. Motivation mechanisms such as rewards in the form of praise, points and power-ups have been included as an integral part of the game in order to stimulate game play (Figure 1) with enemies and a timer to play against providing a competitive element [2] to increase enjoyment.

An AI component has been included based on a defined state machine interoperating with autonomous moving agents [3] in order to provide an additional competitive element. 

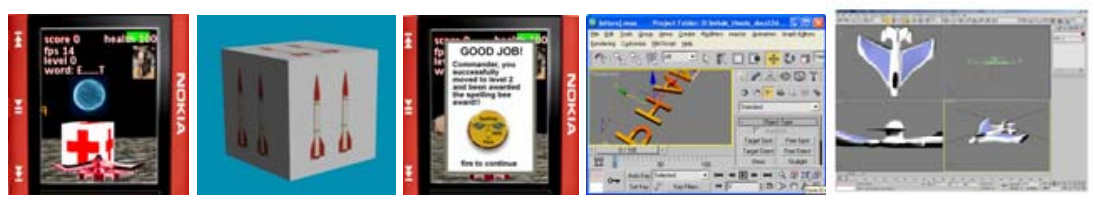

Fig. 1. Health power-up (1), shot power-up (2) , and medal (3) are examples of reward and incentive mechanisms. Letters and 3D models designed in 3DS Max should be pleasing $(4,5)$

Finally, except for the application of motivational mechanisms, significant effort has been made on providing a pleasant $3 \mathrm{D}$ environment since this also plays a role in user satisfaction.

\section{Implementation, Performance and Conclusions}

For MobiSpell implementation, Netbeans Mobility Pack, 3DS Max, Adobe Firefox and the Hi-Corp M3G Plug-in are utilized. Multimedia content is created in a three step process: 2D textures are first created in Adobe Fireworks, 3D Models are then created using the textures, the models are exported into the J2ME Mobile 3D Graphics format and finally imported in J2ME. Extensive use is made of the M3G library to provide the $3 \mathrm{D}$ environment.

The performance of the game was acceptable on a Nokia 6085 cell phone as long as less than two enemy planes and 15 letters existed within the level. Optimization strategies such as using public variables, using a pool design pattern for bullets, and avoiding object oriented encapsulation strategies reduce overhead.

Educational games are effective towards promoting learning - used in conjunction with mobile technology increase all-the-time access to novel learning paradigms. Future work will focus on game extension adding non visual multimedia such as sound (e.g. a spoken word) and optimizing code.

\section{References}

1. Sweetser, P., Wyeth, P.: GameFlow: A Model for Evaluating Player Enjoyment in Games. ACM Computers in Entertainment, Article (2005)

2. Williams, R.B., Clippinger, C.A.: Aggression, competition and computer games: Computer and human opponents. Computers in Human Behavior 18, 495-506 (2002)

3. Bakopoulos, M.: A 3D J2ME Game Utilizing Autonomous Moving Agents. In: Tsekeridou, S., Cheok, A.D., Giannakis, K., Karigiannis, J. (eds.) Proceedings of the 3rd international Conference on Digital Interactive Media in Entertainment and Arts, pp. 513-514. ACM, New York (2008) 